\title{
Utility of an Artificial Intelligence System for Classification of Esophageal Lesions when Simulating its Clinical use
}

\section{Ayaka Tajiri}

Osaka International Cancer Institute

Ryu ISHIHARA ( $\square$ ryu1486@gmail.com )

Osaka International Cancer Institute

\section{Yusuke KATO}

Al Medical Service Inc

\section{Takahiro INOUE}

Osaka International Cancer Institute

\section{Katsunori MATSUEDA}

Osaka International Cancer Institute

\section{Muneaki MIYAKE}

Osaka International Cancer Institute

Kotaro WAKI

Osaka International Cancer Institute

\section{Yusaku SHIMAMOTO}

Osaka International Cancer Institute Hiromu FUKUDA

Osaka International Cancer Institute Noriko MATSUURA

Osaka International Cancer Institute

\section{Satoshi EGAWA}

Osaka Police Hospital

\section{Shinjiro YAMAGUCHI}

Kansai Rosai Hospital

\section{Hideharu OGIYAMA}

Itami City Hospital

Kiyoshi OGISO

JR Osaka Railway Hospital

\section{Tsutomu NISHIDA}

Toyonaka Municipal Hospital

Kenji AOI 
Kaizuka City Hospital

Tomohiro TADA

Al Medical Service Inc

\section{Research Article}

Keywords: Al, Esophageal Neoplasms, Endoscopy, Squamous cell carcinoma

Posted Date: May 26th, 2021

DOI: https://doi.org/10.21203/rs.3.rs-537951/v1

License: (c) (1) This work is licensed under a Creative Commons Attribution 4.0 International License. Read Full License 


\section{Abstract}

\section{Background:}

Previous reports have shown favorable performance of artificial intelligence (AI) systems for diagnosing esophageal squamous cell carcinoma (ESCC) compared with endoscopists. However, these findings don't reflect performance in clinical situations, as endoscopists classify lesions based on both magnified and non-magnified videos, while Al systems often use only a few magnified narrow band imaging (NBI) still images. We evaluated the performance of the Al system in simulated clinical situations.

\section{Methods:}

We used 25,048 images from 1,433 superficial ESCC and 4,746 images from 410 noncancerous esophagi to construct our Al system. For the validation dataset, we took NBI videos of suspected superficial ESCCs. The Al system diagnosis used one magnified still image taken from each video, while 19 endoscopists used whole videos.

\section{Results:}

We used 147 datasets including 83 superficial ESCC and 64 non-ESCC lesions. The accuracy, sensitivity and specificity for the classification of ESCC were, respectively, $80.9 \%, 85.5 \%$, and $75.0 \%$ for the Al system and $69.2 \%, 67.5 \%$, and $71.5 \%$ for the endoscopists. The Al system correctly classified all ESCCs invading the muscularis mucosa or submucosa and $96.8 \%$ of lesions $\geq 20 \mathrm{~mm}$, whereas even the experts misdiagnosed some of them.

\section{Conclusions:}

Our Al system showed higher diagnostic ability for classifying ESCC and non-ESCC than endoscopists. It may provide valuable diagnostic support to endoscopists.

\section{Background:}

Esophageal cancer (EC) is the seventh most common cancer and the sixth most common cause of cancer-related death worldwide (1). Squamous cell carcinoma (SCC) is the most common subtype, and accounts for $80 \%$ of all EC (1). Advanced esophageal squamous cell carcinoma (ESCC) has a poor prognosis, so detecting and diagnosing it at an early stage is important for a favorable outcome (2) (3) (4) (5) (6). Specifically, it is necessary to correctly differentiate cancerous and non-cancerous abnormal lesions detected by esophagogastroduodenoscopy (EGD).

To detect ESCC, narrow band imaging (NBI) and blue laser imaging (BLI), equipment-based imageenhanced endoscopy, are reportedly useful (7) (8) (9) (10). These are advanced, noninvasive optical techniques that enhance visibility of the superficial structure and microvascular pattern of the esophagus (7). Previous studies involving NBI and magnification have shown high diagnostic accuracy for 
esophageal squamous cell carcinoma (7) (11) (12) (13), and they are currently regarded as the standard modality for diagnosing esophageal SCC. However, a previous report also showed that the diagnosis of SCCs by NBI and magnification was liable to interobserver variability, and identification accuracy was not very high (14).

Artificial intelligence (Al) systems have the potential to improve the accuracy of diagnosis by endoscopy. Deep learning, which is typically based on convolutional neural networks, is the mainstay of Al systems, which have shown good performance in visual tasks. This technology has been applied to the diagnosis of GI cancers, including esophageal SCC (15) (16) (17), and previous studies have shown that Al systems have favorable performance in the detection of ESCC (15) (18) (19) (20). In these reports, endoscopists and Al systems used the same magnified still images (15) (18) and video images (19) (20) to diagnose the lesions. In clinical practice, however, endoscopists and Al systems use different methods to make diagnoses. Endoscopists classify lesions comprehensively, based on a variety of images, both magnified and non-magnified images. In contrast, Al systems often use only a few still images for classification, particularly magnified NBI images (15) (18). To evaluate the performance of an Al system as a support tool, it should be evaluated in more realistic situations.

In this study, we compared the performance of our Al system with endoscopists in a situation simulating clinical diagnosis.

\section{Methods:}

\section{Training datasets and image annotation}

We developed a deep learning-based Al system classification of superficial ESCCs. The system was trained with endoscopic images taken on daily EGD. We gathered endoscopic still and video images of pathologically proven superficial ESCC captured at Osaka International Cancer Institute, Fukuoka University Chikushi Hospital, and Niigata University Hospital between December 2005 and June 2019. We also gathered images of noncancerous lesions and normal esophagi taken at Osaka International Cancer Institute between January 2009 and June 2019. Noncancerous lesions included pathologically or endoscopically diagnosed esophagitis, submucosal tumor, vascular abnormality, glycogenic acanthosis, atypical epithelium, and intraepithelial neoplasia. Poor quality images due to bleeding, halation, or defocus were excluded. As in our previous studies (20) (21), still images extracted from videos were used to diversify cancer images in terms of shooting conditions (e.g. various distances, angles, and focus). The endoscopic procedures were carried out using the following equipment: GIF-RQ260Z, GIF-FQ260Z, GIF-Q240Z, GIF-H290Z, GIF-HQ290, GIF-H260Z, GIF-XP290N, GIF-Q260J, or GIF-H290 endoscopes (Olympus, Tokyo, Japan) with the video processors CV260 (Olympus), EVIS LUCERA CV-260/CLV-260, or EVIS LUCERA ELITE CV-290/CLV-290SL (Olympus Medical Systems); or EG-L590ZW, EG-L600ZW, or EGL600ZW7 endoscopes (Fujifilm Co, Tokyo, Japan) and the video endoscopic system LASEREO (Fujifilm Co.). For observations that used the LASEREO system, white-light imaging (WLI) and BLI, which provide images similar to NBI, were used. A black soft hood was equipped on the tip of the endoscope to keep an 
appropriate distance between the tip of the endoscope and esophageal wall during magnified observations. B-mode level 8 for NBI and level 5- 6 for BLI was used for the structure enhancement function. After extracting still images from the videos, our construction dataset included 25,048 images from 1,433 pathologically proven superficial ESCCs and 4,746 images from 410 noncancerous lesions and normal esophagi (Figure 1). These images included those captured by magnified endoscopy (ME) and non-magnified endoscopy (non-ME) with WLI, NBI, and BLI. As in our previous study (20) (21), the images were annotated manually by precisely delineating the boundaries and filling in the areas containing the ESCC or other abnormal lesions. Annotation was conducted by eight endoscopists and all annotated images were reconfirmed by a board-certified trainer (R.I.) at the Japan Gastroenterological Endoscopy Society. While annotating the images, we referred to images captured by various imaging modalities: WLI, NBI/BLI, and chromoendoscopy with and without ME as needed.

\section{Construction of the Al system}

Big Transfer (BiT), a recipe of pre-training networks in computer vision for effective learning of general features, is expected to advance the performance of computer vision models. According to "Big Transfer (BiT): General Visual Representation Learning (https://arxiv.org/abs/1912.11370)", BiT achieved strong performance on over 20 datasets.

For endoscopic images, the new recipe is expected to work well because the pre-trained model already comes with a good understanding of the visual world. All BiT models consist of a vanilla ResNet-v2 architecture which is then customized. Considering the size of the training dataset and validation accuracy, we adopted a BiT-M (ResNet-101x1) for our Al system. This model was pre-trained on the full ImageNet-21k dataset, which is a public dataset containing 14.2 million images and $21 \mathrm{k}$ classes organized by the WordNet hierarchy. At the phase of transfer learning, we trained the model using a BiTHyperRule, which is in the recipe, to select the most important hyperparameters for tuning. We used SGD with an initial learning rate of 0.003 and momentum 0.9 . We fine-tuned the model for 3900 steps with a batch size of 32 . The learning rate was decayed by a factor of 10 at $30 \%, 60 \%$ and $90 \%$ of the training steps. The model was trained on the dataset and validated using the PyTorch deep learning framework, which is one of the most popular and widely used frameworks. For the training dataset, we included endoscopic images with various shooting conditions and resolutions to improve the generalizability of the system. Each image was resized to $512 \times 512$ pixels for optimal analysis.

\section{Video images for the validation dataset}

To create the independent validation datasets, NBI/BLI endoscopic video segments for the diagnoses of suspected superficial esophageal SCCs were taken by eight endoscopists from December 2019 to July 2020 at the Osaka International Cancer Institute. The video, including the continuous diagnostic procedures, consisted of detecting the lesion by non-ME image, coming close to the lesion, and observing the microvascular pattern by ME image. The videos were directly used as validation videos to avoid any 
bias derived from editing. All lesions for validation datasets were pathologically confirmed by biopsy specimens or endoscopic submucosal dissection (ESD) specimens. If the diagnoses of the lesion by biopsy and ESD were different, diagnosis by ESD was adopted. Non-cancerous lesions were also histologically confirmed to have no malignancy, including esophagitis, atypical epithelium or papilloma, or low-grade intraepithelial neoplasia. Subjects with the following were excluded: (1) advanced stage of ESCC, (2) a history of chemotherapy or/and radiation therapy for esophagi, (3) lesions near the esophagogastric junction, (4) lesions near ESD or operation scars, (5) inconclusive diagnosis of cancer by pathology.

Regarding sample size, we initially estimated a minimum of 60 ESCCs for the validation set, based on the sensitivity of $90 \%$ of the Al system in a previous study (20). To maximize study power, we sequentially collected as many ESCCs as possible, more than 60 lesions, from consecutive cases during the period to reduce the $95 \%$ confidence interval to under $20 \%$. Altogether, 147 lesions from 130 patients, including 83 cancers and 64 noncancers, were collected by nine endoscopists for the validation movie dataset.

\section{Evaluation of the Al system and endoscopists}

The trained neural network generated a diagnosis of esophageal SCC or noncancerous lesions, such as abnormal vessels or esophagitis, with a continuous value between 0 and 1, corresponding to the probability of that diagnosis. We took one still ME image, just like our daily practice, from the validation video dataset. These still images (validation still image dataset) were judged by the Al system as ESCC or non-ESCC.

We invited 19 endoscopists with varying years of experience from six centers to interpret the validation dataset. Thirteen of them were board-certified specialists at the Japan Gastroenterological Endoscopy Society (experts). The median years of experience of endoscopy of all endoscopists was 12 (range: 2-25 years) and the median number of EGDs they had performed was 6000 (range: 500-25,000), and for the experts only were 15 years (range: 9-25) and 10,000 (range: 4000-25,000). They had been diagnosing gastrointestinal cancers, including superficial ESCC, in their daily practice. They interpreted the validation video dataset, and the validation still image dataset if needed, and classified them to ESCC or non-ESCC.

\section{Statistical analysis}

The main outcome measures were diagnostic accuracy, sensitivity and specificity for superficial ESCC. These parameters were calculated as follows: Accuracy = correctly diagnosed lesions/total lesions; Sensitivity = (the number of correctly detected superficial ESCC) / (the number of total superficial ESCC); Specificity $=$ (the number of correctly diagnosed noncancerous lesions or normal esophagus videos) / (the number of total noncancers or normal esophagus videos). Results are shown as averages (arithmetic mean) and range. All analyses were performed on a personal computer using the EZR software package, version 1.27 (Saitama Medical Center, Jichi Medical University, Tochigi, Japan). 


\section{Ethics approval}

This study was approved by the ethics committee of Osaka International Cancer Institute (no. 18149-5). We confirmed that all experiments were performed in accordance with relevant guidelines and regulations. The movies of endoscopy for constructing the Al system was retrospectively collected, and for validating the Al system was collected in anonymized form and non-interventional way, so informed consent was obtained from all patients by opt-out.

\section{Results:}

\section{Details of validation datasets and endoscopist}

A total of 147 videos were taken by eight endoscopists from December 2019 to July 2020. After excluding 31 videos based on the exclusion criteria, 83 ESCC video and 64 non-ESCC videos were included in the validation dataset (Figure 1). The videos were between 4 and 29 seconds long, and details of included lesions are shown in Table 1.

\begin{tabular}{|c|c|}
\hline \multicolumn{2}{|l|}{ Table 1} \\
\hline \multicolumn{2}{|c|}{ Summary of the validation dataset } \\
\hline ESCC & 83 \\
\hline Tumor size in $\mathrm{mm},<5 / 5 \sim 10 / 10 \sim 20 / 20<$ & $3 / 20 / 32 / 28$ \\
\hline 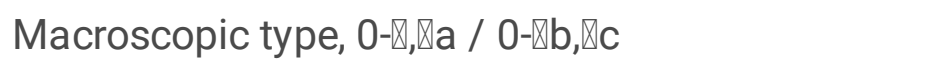 & $7 / 76$ \\
\hline Depth of tumor*, EP-LPM / MM / SM1 / SM2-3 & $52 / 12 / 2 / 7$ \\
\hline Non-cancerous lesions & 64 \\
\hline lesion size in $\mathrm{mm},<5 / 5 \sim 10 / 10 \sim 20 / 20<$ & $14 / 31 / 16 / 3$ \\
\hline LGIN / Atypical / Esophagitis / Papilloma / Others & $9 / 10 / 24 / 1 / 20$ \\
\hline \multicolumn{2}{|l|}{ ESCC: esophageal squamous cell carcinoma } \\
\hline \multicolumn{2}{|c|}{ EP: epithelium, LPM: lamina propria, MM: muscularis mucosa, SM: submucosa } \\
\hline \multicolumn{2}{|l|}{ LGIN: low-grade intraepithelial neoplasia } \\
\hline *Ten ESCCs diagnosed by biopsy were excluded frc & sis of invasion depth. \\
\hline
\end{tabular}

\section{Performance of the Al system versus the endoscopists}


The Al system diagnosed 71 of 83 SCCs (85.5\%) as cancers and 48 of 64 noncancers $(75.0 \%)$ as noncancers. The diagnostic performance of the Al system and the endoscopists are shown in Table 2. Accuracy, sensitivity and specificity of the Al system were all superior to that of the endoscopists, even that of the experts.

\begin{tabular}{|c|c|c|c|c|}
\hline \multicolumn{5}{|c|}{ Table 2} \\
\hline \multicolumn{5}{|c|}{ Diagnostic performance of the Al system and the endoscopists } \\
\hline & Al system & All endoscopists $(n=19)$ & Experts $(n=13)$ & Non-experts $(n=6)$ \\
\hline & & (Average[range]) & (Average[range]) & (Average[range]) \\
\hline Accuracy & $80.9 \%(119 / 147)$ & $69.2 \%[59.2-77.6 \%]$ & $69.9 \%[59.9-77.6]$ & $67.9 \%[59.2-76.2]$ \\
\hline Sensitivity & $85.5 \rrbracket(71 / 83)$ & $67.5 \%[37.3-85.5]$ & $68.5 \%[37.3-85.5]$ & $65.5 \%[51.8-81.9]$ \\
\hline Specificity & $75.0 \%(48 / 64)$ & $71.5 \%[32.8-96.9]$ & $71.6 \%[32.8-96.9]$ & $71.1 \otimes[46.8-90.6]$ \\
\hline Al: artificia & telligence & & & \\
\hline
\end{tabular}

\section{Subgroup analyses by pathological diagnosis and size of the lesion}

Table 3 shows the accuracy of the Al system and the endoscopists with respect to pathological diagnosis. The Al system showed better accuracy than the endoscopists in all categories, especially in ESCCs. Moreover, the Al system correctly classified all ESCCs that invaded the muscularis mucosa or submucosa, whereas even the experts misdiagnosed some of these cancers.

Similarly, Table 3 shows the accuracy with respect to lesion size. The Al system also showed better accuracy than the endoscopists in these categories. In addition, the Al system correctly classified $96.8 \%$ of lesions $\geq 20 \mathrm{~mm}$. 


\section{Table 3}

Accuracy of the Al system and endoscopist by pathological diagnosis and size of lesion

Al system

Endoscopists*

all Experts Non-experts

By pathological diagnosis

\begin{tabular}{lllll} 
ESCC :83 cases & $85.5 \%(71 / 83)$ & $67.5 \%$ & $68.5 \rrbracket$ & $65.5 \rrbracket$ \\
pEP/LPM : 52 cases & $77.4 \%(41 / 52)$ & $57.2 \%$ & $57.9 \%$ & $55.7 \%$ \\
pMM/SM1/SM2 : 21 cases & $100 \%(21 / 21)$ & $89.0 \%$ & $89.7 \%$ & $87.3 \%$ \\
\hline Non-ESCC :64 cases & $75 \%(48 / 64)$ & $71.5 \%$ & $71.6 \%$ & $71.1 \%$ \\
LGIN/ atypical : 19 cases & $68.4 \rrbracket(13 / 19)$ & $64.8 \%$ & $64.0 \%$ & $66.7 \%$ \\
Esophagitis/papilloma/others: 45 cases & $77.8 \%(35 / 45)$ & $74.3 \%$ & $74.9 \%$ & $73.0 \%$ \\
\hline By size of the lesions & & & & \\
\hline$<10 \mathrm{~mm}: 68$ cases & $72.1 \%(49 / 68)$ & $68.0 \%$ & $67.6 \%$ & $68.9 \%$ \\
\hline$\geq 10 \mathrm{~mm}: 79$ cases & $88.6 \%(70 / 79)$ & $70.3 \%$ & $71.8 \%$ & $67.1 \%$ \\
$\geq 20 \mathrm{~mm}: 31$ cases & $96.8 \%(30 / 31)$ & $82.7 \rrbracket$ & $84.1 \rrbracket$ & $79.6 \rrbracket$
\end{tabular}

Al: artificial intelligence, ESCC: esophageal squamous cell carcinoma

EP: epithelium, LPM: lamina propria, MM: muscularis mucosa, SM: submucosa

LGIN: low-grade intraepithelial neoplasia

* Percentages shown are averages.

\section{The characteristics of the lesion that The Al system had better accuracy than the endoscopist}

Table 4 shows the characteristics of nine lesions that the Al system classified correctly but less than $30 \%$ of the endoscopists could. Eight of them were cancerous, and six of nine lesions were $10 \mathrm{~mm}$ or more in size. It revealed that the Al system could correctly classify ESCCs that the many endoscopists incorrectly judged as non-ESCCs, and it might have more advantage for larger lesions.

For example, Figure 2 shows the ESCC cases that the Al system classified correctly although more than $70 \%$ of the endoscopists misdiagnosed them as non-ESCC. These lesions showed faint background 
coloration and slightly dilatated intrapapillary capillary loops. They had been treated by ESD, and histologically diagnosed as ESCC.

\section{Table 4}

Lesions correctly classified by the Al system but by less than $30 \%$ of the endoscopists

\begin{tabular}{|llll|}
\hline & ESCC & non-ESCC & Total \\
\hline$<10 \mathrm{~mm}$ & 3 & 0 & $3(33.3 \%)$ \\
$10 \mathrm{~mm} \leq$ & 5 & 1 & $6(66.7 \%)$ \\
\hline Total & $8(88.9 \%)$ & $1(11.1 \%)$ & 9 \\
\hline ESCC: esophageal squamous cell carcinoma & \\
\hline
\end{tabular}

These lesions showed fainter background coloration and slightly dilatated intrapapillary capillary loops.

\section{Discussion:}

In this study, we confirmed the high accuracy of our Al system, which was superior to the endoscopists, including the experts. Among various diagnostic parameters, our Al system showed a sensitivity that was about $20 \%$ higher than the experts. In addition, our Al correctly diagnosed eight cancers that were not correctly diagnosed by the majority $(>70 \%)$ of endoscopists. Because of its high sensitivity, our Al system could reduce the number of cancers being overlooked.

In subgroup analysis divided by lesions' size and cancer invasion depth, our Al showed better performance in all subgroups. In particular, the performance was better in clinically significant lesions such as $\mathrm{pMM} / \mathrm{SM} 1 / \mathrm{SM} 2$ cancers and lesions $\geq 10 \mathrm{~mm}$. Although final diagnosis is made by confirming pathological results of biopsy specimens, accurate endoscopic diagnosis is important to avoid overlooking cancers and to make appropriate clinical decisions.

Classification of lesions by the Al system can be conducted using video or still images. Most Al systems use still images for classification (15) (18) (22) and we are also planning to introduce a similar system to the practice. Therefore, in this study, we used still images for the evaluation of our Al system. Conversely, endoscopists can classify lesions based on magnified and non-magnified observations. We therefore used video images of non-magnified and magnified observation for the evaluation of endoscopists. We consider that our study simulates the use of the Al system in clinical practice and reflects its performance in comparison with endoscopists.

From these results, our Al system would be a useful tool for supporting diagnosis, as it has a higher sensitivity and specificity for cancer than general endoscopists. In addition, considering that the accuracy of endoscopic diagnosis had been reported to be comparable with that of biopsy diagnosis (23), our Al system might be implemented as an optical biopsy with a high quality of diagnosis. 
This study has several limitations. First, we excluded four cases from the validation dataset where the pathological diagnosis was inconclusive because we could not determine whether such lesions should be regarded as cancer in the analysis. Second, esophago-gastric junctional cancers (E/J cancer), including Barrett's adenocarcinoma, were not included in this study. Because endoscopic findings of $\mathrm{E} / \mathrm{J}$ cancer are quite different from ESCC, creation of a specialized Al system for E/J cancer is necessary. In this study we focused on the evaluation of ESCC.

In conclusion, our Al system showed higher accuracy than endoscopists for classifying ESCC and noncancerous lesions by ME in a situation simulating clinical use of the system. This system may therefore provide valuable support for endoscopists.

\section{Declarations}

\section{Acknowledgements:}

We thank R. Takeda (Osaka Police Hospital), A. Shimayosi (Osaka Police Hospital), Y. Morita (Osaka Police Hospital), T. Hirao (Osaka Police Hospital), T. Ohta (Kansai Rosai Hospital), Y. Adachi (Kansai Rosai Hospital), K. Watsuji (Kansai Rosai Hospital), T. Sanomura (Itami City Hospital), T. Iwasaki (Itami City Hospital), K. Uejima (JR Osaka Railway Hospital), K. Ikeda (JR Osaka Railway Hospital), M. Yamamoto (Toyonaka Municipal Hospital), and S. Satake (Kaizuka City Hospital) for acting as the endoscopists. We also thank Peter Morgan, PhD, from Edanz Group (https://en-authorservices.edanz.com/ac) for editing a draft of this manuscript.

\section{Author contributions:}

A. T: collecting endoscopic images for constructing Al system, interpreting the data and drafting the manuscript

R. I: conception and design, collecting endoscopic images for constructing Al system, critical revision of the article for important intellectual content, and final approval of the article

Y. K, T. T: constructing and mechanical management the Al system

T. I, K. M, M. M, K. W, Y. S, H. F, N. M: collecting endoscopic images for constructing Al system

S. E, S. Y, H. O, K. O, T. N, K. A: having the role to check the Al system

\section{Financial support:}

There is no financial sponsor for this study. 


\section{Potential competing interests:}

T.T is a shareholder in Al Medical Service Inc. All other authors have no financial relationships to declare.

\section{References}

1. Bray, F. et al . Global cancer statistics 2018: GLOBOCAN estimates of incidence and mortality worldwide for 36 cancers in 185 countries. CA Cancer J Clin. 68(6), 394-424 (2018).

2. Katada, C. et al. Clinical outcome after endoscopic mucosal resection for esophageal squamous cell carcinoma invading the muscularis mucosae--a multicenter retrospective cohort study. Endoscopy. 39(9), 779-83 (2007).

3. Kodama, M. et al. Treatment of superficial cancer of the esophagus: a summary of responses to a questionnaire on superficial cancer of the esophagus in Japan. Surgery. 123(4), 432-9 (1998).

4. Shimizu, Y. et al. Long-term outcome after endoscopic mucosal resection in patients with esophageal squamous cell carcinoma invading the muscularis mucosae or deeper. Gastrointest Endosc. 56(3), 387-90 (2002).

5. Yamamoto, S. et al. Comparison between definitive chemoradiotherapy and esophagectomy in patients with clinical stage I esophageal squamous cell carcinoma. Am J Gastroenterol. 106(6), 1048-54 (2011).

6. Yamashina, T. et al. Long-term outcome and metastatic risk after endoscopic resection of superficial esophageal squamous cell carcinoma. Am J Gastroenterol. 108(4), 544-51 (2013).

7. Muto, M. et al. Early detection of superficial squamous cell carcinoma in the head and neck region and esophagus by narrow band imaging: a multicenter randomized controlled trial. J Clin Oncol. 28(9), 1566-72 (2010).

8. Gono, K. et al. Appearance of enhanced tissue features in narrow-band endoscopic imaging. $\mathrm{J}$ Biomed Opt. 9(3), 568-77 (2004).

9. Ishihara, R. et al. Prospective evaluation of narrow-band imaging endoscopy for screening of esophageal squamous mucosal high-grade neoplasia in experienced and less experienced endoscopists. Dis Esophagus. 23(6), 480-6 (2010).

10. Tomie, A. et al. Blue Laser Imaging-Bright Improves Endoscopic Recognition of Superficial Esophageal Squamous Cell Carcinoma. Gastroenterol Res Pract.2016:6140854 (2016).

11. Yoshida, T. et al. Narrow-band imaging system with magnifying endoscopy for superficial esophageal lesions. Gastrointest Endosc. 59(2), 288-95 (2009).

12. Takenaka, R. et al. Narrow-band imaging provides reliable screening for esophageal malignancy in patients with head and neck cancers. Am J Gastroenterol. 104(12), 2942-8 (2009).

13. Ishihara, R. et al. Significance of each narrow-band imaging finding in diagnosing squamous mucosal high-grade neoplasia of the esophagus. J Gastroenterol Hepatol. 25(8), 1410-5 (2010). 
14. Ohmori, M. et al. Endoscopic detection and differentiation of esophageal lesions using a deep neural network. Gastrointest Endosc. 91(2):301-9.e1 (2019).

15. Horie, Y. et al. Diagnostic outcomes of esophageal cancer by artificial intelligence using convolutional neural networks. Gastrointest Endosc. 89, 25-32 (2019).

16. Hirasawa, T. et al. Application of artificial intelligence using a convolutional neural network for detecting gastric cancer in endoscopic images. Gastric Cancer. 21(4), 653-60 (2018).

17. Alaskar, H. et al. Application of Convolutional Neural Networks for Automated Ulcer Detection in Wireless Capsule Endoscopy Images. Sensors (Basel).19(6), 2019.

18. Ohmori, M. et al. Endoscopic detection and differentiation of esophageal lesions using a deep neural network. Gastrointest Endosc. 91, 301-9 (2020).

19. Guo, L. et al. Real-time automated diagnosis of precancerous lesions and early esophageal squamous cell carcinoma using a deep learning model (with videos). Gastrointest Endosc. 91, 41-51 (2020).

20. Fukuda, H. et al. Comparison of performances of artificial intelligence versus expert endoscopists for real-time assisted diagnosis of esophageal squamous cell carcinoma (with video). Gastrointest Endosc. 92, 848-55 (2020).

21. Waki, K. et al. Usefulness of an artificial intelligence system for the detection of esophageal squamous cell carcinoma evaluated with videos simulating overlooking situation. Dig Endosc. doi: 10.1111/den.13934 (2021).

22. Mori, Y. et al. Real-Time Use of Artificial Intelligence in Identification of Diminutive Polyps During Colonoscopy: A Prospective Study. Ann Intern Med. 169, 357-66 (2018).

23. Nagai, K. et al. Endoscopic optical diagnosis provides high diagnostic accuracy of esophageal squamous cell carcinoma. BMC Gastroenterol. 14, 141 (2014).

\section{Figures}




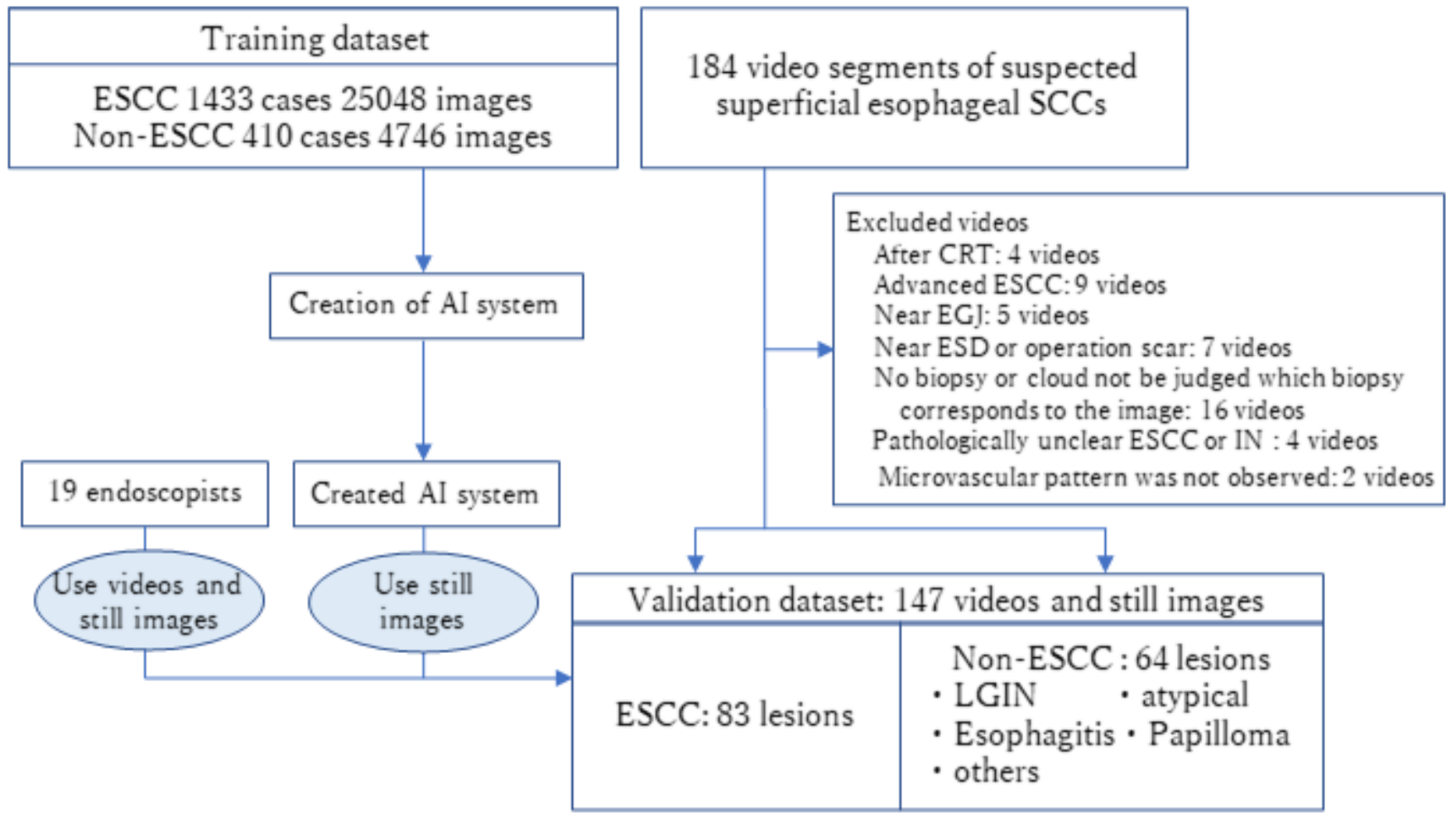

\section{Figure 1}

Flowchart of construction of Al and validation dataset Al: artificial intelligence, ESCC: esophageal squamous cell carcinoma CRT: chemoradiotherapy, EGJ: esophagogastric junction, ESD: endoscopic submucosal dissection IN: intraepithelial neoplasia, LGIN: low-grade intraepithelial neoplasia

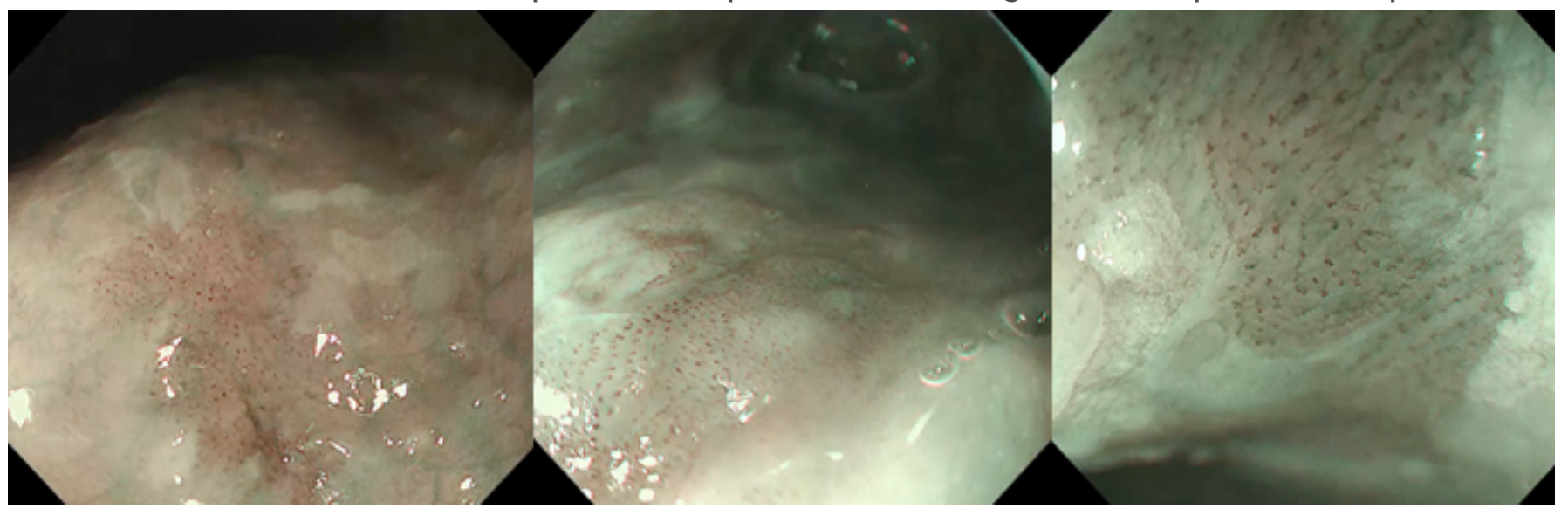

Figure 2

ESCCs cases correctly classified by the Al system but misdiagnosed as non-ESCCs by more than $70 \%$ of the endoscopists These lesions showed fainter background coloration and slightly dilatated intrapapillary capillary loops.

\section{Supplementary Files}


This is a list of supplementary files associated with this preprint. Click to download.

- tablessr.docx 